\title{
Bipartite Interaction between Neurofibromatosis Type I Protein (Neurofibromin) and Syndecan Transmembrane Heparan Sulfate Proteoglycans
}

\author{
Yi-Ping Hsueh, ${ }^{1,2}$ Anne M. Roberts, ${ }^{3}$ Manuela Volta,, ${ }^{3}$ Morgan Sheng, ${ }^{2}$ and Roland G. Roberts ${ }^{3}$ \\ 1/nstitute of Molecular Biology, Academia Sinica, Taipei, Taiwan, 115, Republic of China, ${ }^{2 H o w a r d ~ H u g h e s ~ M e d i c a l ~}$ \\ Institute and Department of Neurobiology, Massachusetts General Hospital and Harvard Medical School, Boston, \\ Massachusetts 02114, and 'Division of Medical and Molecular Genetics, Guy's, King's, and St. Thomas's Medical \\ School, London SE1 9RT, United Kingdom
}

The neurofibromatosis type 1 (NF1) gene encodes a large tumor suppressor protein (neurofibromin). Although it is known to possess Ras GTPase-activating protein (GAP) activity, the cellular role of neurofibromin remains unclear. Here we used yeast two-hybrid screening to identify neurofibromin-interacting proteins. Syndecan-2, a transmembrane heparan sulfate proteoglycan (HSPG), was isolated as a binding partner for two distinct regions of the neurofibromin protein. We subsequently found that neurofibromin can bind all four mammalian syndecans. NF1 interaction requires the transmembrane domain and a membrane-proximal region of the cytoplasmic tail of syndecan, but not the $\mathrm{C}$ terminus of syndecan known to bind to

Neurofibromatosis type I (NF1) is a common dominantly inherited neurocutaneous disorder characterized by benign and malignant tumors of the nervous system. The disease phenotype seems to be mainly a manifestation of abnormal proliferation and/or differentiation in cells of neural crest origin. The NF1 gene is widely expressed in many tissues but at particularly high levels in the CNS and peripheral nervous system (PNS; Daston et al., 1992; Nordlund et al., 1993). NF1 encodes a large protein of 2818 amino acids (aa) known as neurofibromin; most germline mutations in the NF1 gene are predicted to result in a truncated and probably nonf unctional protein. A central 350-amino-acid region of neurofibromin (the GAP-related domain or GRD) is homologous to known GTPase-activating proteins (GAPs). This GRD has been shown to act as a GAP for the Ras family of small GTPases that regulate cell growth and differentiation (Ballester et al., 1990; Martin et al., 1990). Homozygous mice with targeted disruption of the NF1 gene show lethal developmental abnormalities, whereas heterozygotes are predisposed to the development of various tumor types (Brannan et al., 1994; Jacks et al., 1994; Cichowski et al., 1999; Vogel et al., 1999). It is assumed that neurofibromin acts as a tumor suppressor by inhibiting the activ-

\footnotetext{
Received Dec. 8, 2000; revised March 5, 2001; accepted March 7, 2001.

This work was supported by the National Neurofibromatosis Foundation Young Investigator Award (Y.-P.H.), Academia Sinica, Taiwan (Y.-P.H.), the Special Trustees of Guy's and St. Thomas's Hospitals (A.M.R), and the Generation Trust (M.V.). R.G.R. was the recipient of a Wellcome International Fellowship (045735). M.S. is Assistant Investigator of the Howard Hughes Medical Institute.

Correspondence should be addressed to Morgan Sheng, Howard Hughes Medical Institute, Massachusetts General Hospital (Wellman 423), 50 Blossom Street, Boston, MA 02114. E-mail: sheng@helix.mgh.harvard.edu. Correspondence also may be addressed to Yi-Ping Hsueh, Institute of Molecular Biology, Academia Sinica, Taipei, Taiwan, 115, Republic of China. E-mail: yph@gate.sinica.edu.tw. Copyright (C) 2001 Society for Neuroscience $0270-6474 / 01 / 213764-07 \$ 15.00 / 0$
}

CASK, a membrane-associated guanylate kinase (MAGUK). Neurofibromin, syndecans, and CASK have overlapping subcellular distributions in axons and synapses of neurons, as shown by biochemical fractionation and immunostaining. Moreover, neurofibromin exists in a complex with syndecan and CASK in vivo, as evidenced by their coimmunoprecipitation from rat brain. Our findings suggest that interaction with different members of the syndecan family may be a mechanism for localizing neurofibromin to specialized domains of the plasma membrane.

Key words: NF1; RasGAP; postsynaptic density; axon; yeast two-hybrid analysis; CASK/LIN-2

ity of Ras. In addition, Drosophila neurofibromin has been shown to be a regulator of the CAMP pathway and to be required for learning (Guo et al., 1997, 2000) However, the precise cellular roles of neurofibromin remain unclear. To gain potential clues to neurofibromin function, we used the yeast two-hybrid system to search for neurofibromin-interacting proteins. Here, we report that syndecans bind specifically to neurofibromin.

The syndecan family of transmembrane proteins (syndecans 1-4) constitutes a major class of heparan sulfate proteoglycans (HSPGs) on the cell surface (for review, see Bernfield et al., 1992; David, 1993; Carey, 1997). By binding to a variety of secreted growth/differentiation factors at the cell surface (e.g., fibroblast growth factor, FGF), syndecan HSPGs are believed to function as coreceptors in many receptor tyrosine kinase signaling pathways (Schlessinger et al., 1995; Perrimon and Bernfield, 2000). In addition, syndecans bind to extracellular matrix (ECM) proteins such as laminin and fibronectin and are implicated in cell matrix adhesion, cell movement, and tissue morphogenesis.

Extracellular domains of syndecans show little homology, but their short cytoplasmic domains ( $\sim 30$ amino acids) are highly conserved. The C-terminal four amino acids (-EFYA) shared by all syndecans interact with CASK (the mammalian LIN-2 homolog) (Cohen et al., 1998; Hsueh et al., 1998). In Caenorhabditis elegans the CASK homolog LIN-2 associates with two other PDZ-containing proteins, LIN-7 and LIN-10 (Kaech et al., 1998), and all three are required for proper subcellular localization of the epidermal growth factor (EGF) receptor (Kim, 1997). A homologous ternary complex also exists in mammalian brain (Butz et al., 1998). The mammalian LIN-2/LIN-7/LIN-10 complex probably also is involved in subcellular targeting of receptors and cytoskeletal/signaling proteins to specific plasmalemmal do- 
mains. In this report we show that neurofibromin is associated with syndecans and CASK in brain, thus providing a potential mechanism for localizing neurofibromin in a molecular microenvironment with specific cell surface receptors and other signaling molecules.

\section{MATERIALS AND METHODS}

Plasmid construction. Ten segments spanning the human neurofibromin coding region were amplified by using KlenTaq Advantage DNA polymerase with Marathon Ready human whole brain cDNA (Clontech, Palo Alto, CA) according to the manufacturer's directions. The primers (sequences available on request) were engineered to contain EcoRI restriction sites. After purification and digestion these fragments were ligated into EcoRI-digested pBHA and validated by sequencing. Interstitial deletions of the syndecan-2 coding region were generated by overlap extension that used chimeric primers (sequences available on request). To construct synd-2C and $\mathrm{C}$-terminal deletion mutants of syndecan-2 for yeast two-hybrid experiments, we PCR-amplified and subcloned the desired sequences of syndecan-2 into an EcoRI site of vector pGAD10 (Clontech). For construction of GST fusion proteins, the coding regions of rat syndecan-2 from amino acid residue 33 to the end (residue 211 for GST-synd-2 or residue 145 for GST-synd-2 $\Delta 65$ ) were subcloned into an EcoRI site of vector pGEX-4T-1 (Pharmacia, Piscataway, NJ).

Antibodies. Rabbit polyclonal antibodies NF1GRP(D) and NF1GRP(N) were obtained from Santa Cruz (Santa Cruz, CA). Rabbit antibodies Syn-2C and Syn-3C that recognize the cytoplasmic domain of syndecan-2 and syndecan-3, respectively, have been described previously (Hsueh et al., 1998; Hsueh and Sheng, 1999). The CASK monoclonal antibody also has been described (Hsueh and Sheng, 1999).

Yeast two-hybrid analysis. Yeast two-hybrid analysis was done by using the L40 yeast strain harboring reporter genes HIS3 and LacZ under the control of upstream LexA binding sites, as described previously (Niethammer and Sheng, 1998). Bait constructs for each segment of the neurofibromin coding region were cotransfected into L40 yeast host with pGAD10-cloned cDNA libraries from adult and fetal human brain (Clontech). This yielded $\sim 4 \times 10^{-6}$ transformants per bait-library combination. Colonies growing on histidine-deficient medium (supplemented with $2.5 \mathrm{~mm} 3$-aminotriazole) and yielding a blue color with $\mathrm{X}$-gal were selected for plasmid recovery.

For liquid culture assay $4 \mathrm{ml}$ of fresh yeast extract, peptone, and dextrose (YPD) medium was added to $1 \mathrm{ml}$ of overnight culture. The cultures were incubated at $30^{\circ} \mathrm{C}$ for a further $3.5 \mathrm{hr}$ with shaking to reach $\mathrm{OD}_{600}=0.5-0.6$. The exact $\mathrm{OD}_{600}$ was recorded at the time of harvesting of the cells. Then $1 \mathrm{ml}$ of culture was transferred to a microcentrif uge tube, washed once with $\mathrm{Z}$ buffer, and resuspended in $0.1 \mathrm{ml} \mathrm{Z}$ buffer. Three freeze/thaw cycles were performed by using liquid nitrogen and a $37^{\circ} \mathrm{C}$ water bath to break down yeast cell walls. Then $0.7 \mathrm{ml}$ of $\mathrm{Z}$ buffer containing $0.0027 \%(\mathrm{v} / \mathrm{v}) \beta$-mercaptoethanol and $0.16 \mathrm{ml}$ of $4 \mathrm{mg} / \mathrm{ml}$ $o$-nitrophenyl- $\beta$-D-galactopyranoside in $\mathrm{Z}$ buffer was added to each tube. After development of a yellow color, $0.4 \mathrm{ml}$ of $1 \mathrm{M} \mathrm{Na}_{2} \mathrm{CO}_{3}$ was added to the tubes. Incubation time was recorded in minutes. After centrif ugation at $14,000 \mathrm{rpm}$ for $10 \mathrm{~min}$, the $\mathrm{OD}_{420}$ of the supernatant was measured. $\mathrm{Z}$ buffer without yeast cells was used as a blank to calibrate the spectrophotometer. $\beta$-galactosidase units were calculated as $\left(1000 \times \mathrm{OD}_{420}\right) /$ (incubation time $\times \mathrm{OD}_{600}$ ).

Subcellular fractionation of rat brain extracts. Subcellular fractions of adult rat brain were prepared as described previously (Huttner et al., 1983). Briefly, rat brain Dounce homogenate was centrifuged at $1000 \times$ $g$ to remove nuclei and other large debris (pelleted in P1). The supernatant was centrifuged at $10,000 \times g$ to obtain a crude synaptosomal fraction (P2), which subsequently was lysed with hypotonic buffer and centrifuged at $25,000 \times g$ to pellet a lysed synaptosomal membrane fraction (LP1). Then the supernatant (LS1) was centrifuged at $165,000 \times$ $g$ to obtain a crude synaptic vesicle fraction (LP2) and soluble fraction (LS2). The supernatant above the P2 fraction was centrifuged at $165,000 \times g$ to obtain a soluble fraction (S3) and a light membrane fraction (P3). For developmental studies the P2 fractions were collected from the rats at the ages of embryonic day 14 (E14) and E18 and postnatal day 3 (P3), P7, P15, P22, and P42.

GST pulldown assay and coimmunoprecipitation. One-week-old rat brains were minced and homogenized by Dounce homogenizer in PBS. Total homogenate was solubilized further with $1 \%$ Triton $\mathrm{X}-100$ at $4^{\circ} \mathrm{C}$ for $1 \mathrm{hr}$. For GST pulldown assay the extract was diluted with sufficient
PBS to reduce the final concentration of Triton X-100 to $0.25 \%$. Insoluble material was pelleted by centrifugation at $37,000 \times g$ for $1 \mathrm{hr}$. Then the supernatant was incubated with glutathione agarose prebound with GST fusion protein for $\sim 4 \mathrm{hr}$ at $4^{\circ} \mathrm{C}$. Unbound proteins were removed by extensive washing, and the precipitates were analyzed by immunoblotting. Immunoprecipitation of Triton X-100-solublized extract was performed as described previously (Hsueh and Sheng, 1999), except that antibody NF1GRP(N) (Santa Cruz) was used for immunoprecipitation and antibody NF1GRP(D) (Santa Cruz) was used in immunoblotting.

Immunofluorescence staining. Double-label immunofluorescence that used rat brain with two antibodies raised in the same species was performed as described previously (Hsueh et al., 1998). The procedure was modified slightly for the staining of cultured hippocampal neurons. Briefly, cultured neurons were washed three times with PBS and then fixed with $4 \%$ formaldehyde and $4 \%$ sucrose in PBS for $10 \mathrm{~min}$. The cells were treated further with $3 \% \mathrm{H}_{2} \mathrm{O}_{2}$ in methanol at $-20^{\circ} \mathrm{C}$ for $15 \mathrm{~min}$. After 30 min of incubation in PBS with $0.5 \%$ blocking reagent (TSA fluorescence kit, NEN Life Science Products, Boston, MA) the cells were incubated with $50 \mathrm{ng} / \mathrm{ml} \mathrm{Syn-2C}$ or Syn-3C antibodies for $2 \mathrm{hr}$ at room temperature or overnight at $4^{\circ} \mathrm{C}$. (This concentration of primary antibody gives no detectable signal with conventional secondary antibody immunofluorescence protocols.) After being washed three times with PBST (PBS containing 0.05\% Tween 20), the cells were incubated with a 1:1000 dilution of biotinylated anti-rabbit antibody (NEN Life Science Products) at room temperature for $1 \mathrm{hr}$ and were incubated further with a 1:200 dilution of streptavidin-conjugated horseradish peroxidase (TSA fluorescence kit, NEN Life Science Products) for $30 \mathrm{~min}$. Finally, Cy3conjugated tyramide (TSA fluorescence kit, NEN Life Science Products) was applied at a 1:100 dilution in amplification diluent (TSA fluorescence kit, NEN Life Science Products) for $10 \mathrm{~min}$ to visualize Syn-2C or Syn-3C staining. After being washed three times with PBST, these cells were processed for conventional neurofibromin immunofluorescence staining with FITC-conjugated secondary antibody as described previously (Hsueh and Sheng, 1999). The results were analyzed with a confocal microscope (LSM510, Carl Zeiss, Thornwood, NY) with a narrow bandpass filter, and digitized images were processed for publication with Adobe Photoshop (Mountainview, CA).

\section{RESULTS}

\section{Identification of syndecan-2 as a neurofibromin-binding protein}

To identify neurofibromin-interacting proteins, we divided the neurofibromin coding region into 10 segments, each of which was used as bait in yeast two-hybrid screens of human brain cDNA libraries. Specific positives were isolated with three regions of neurofibromin. The N-terminal region (aa 1-135) interacts with a novel protein with no obvious similarities to known proteins. Remarkably, two other regions of neurofibromin, aa 1356-1562 (in the GRD, hereafter called the J fragment) and aa 2616-2812 (near the $\mathrm{C}$ terminus, hereafter called the $\mathrm{P}$ fragment), "fished out" the same interacting clones from the library (Fig. $1 A$ ). The isolated clones contained amino acid residues $87-211$ or $97-211$ of human syndecan-2, which includes part of the N-terminal extracellular domain, the entire transmembrane, and cytoplasmic domains of syndecan-2. None of the other regions of neurofibromin interacted with syndecan-2 in yeast two-hybrid analysis (data not shown). To narrow down the syndecan-2 binding site on neurofibromin, we further divided $\mathrm{J}$ and $\mathrm{P}$ regions into $\mathrm{N}$ - and C-terminal fragments ( $\mathrm{Jn} / \mathrm{Jc}$ and $\mathrm{Pn} / \mathrm{Pc}$, respectively). By yeast two-hybrid assays either Jn (aa 1357-1473) or Pn (aa 2619-2719) regions of neurofibromin were sufficient to interact with syndecan-2 (Fig. 1A). Thus, two separate parts of neurofibromin can interact with syndecan-2.

To examine the interaction of syndecan- 2 and full-length neurofibromin, we performed GST pulldown assays from rat brain extract. Neurofibromin was precipitated specifically by a GST fusion of syndecan-2 (amino acid residue 33-211) containing part of the extracellular domain, plus complete transmembrane and 
A.

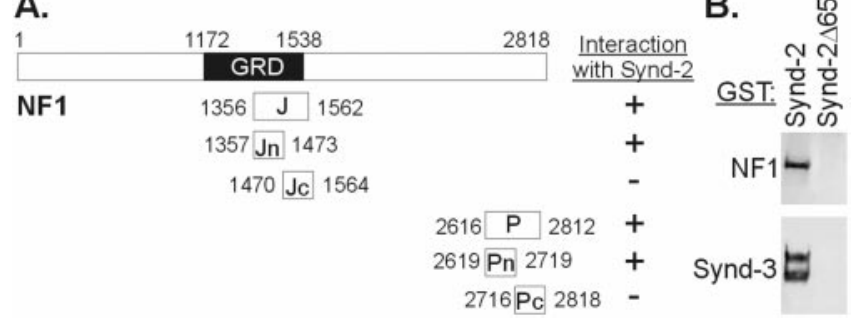

Figure 1. Bipartite interaction of neurofibromin and syndecan-2. A, Neurofibromin is depicted schematically at the top; the $\mathrm{J}$ and $\mathrm{P}$ regions used as baits in yeast two-hybrid screens are aligned below. GRD, GAPrelated domain. Interactions with human syndecan- 2 are summarized, based on induction of the reporter gene $\beta$-galactosidase in yeast twohybrid assays. $B$, GST pulldown assays from brain. Triton X-100 rat brain extract was incubated with glutathione agarose charged with GSTsyndecan-2 (Synd-2) or GST-syndecan-2 mutant lacking the last 65 amino acids (Synd-2 $\Delta 65)$. The precipitates were analyzed via immunoblotting by using neurofibromin (NF1) and syndecan-3 (Synd-3) antibodies.

cytoplasmic domains of rat syndecan-2 (Fig. $1 B$; data not shown). GST-syndecan-2 $\Delta 65$, which lacks the C-terminal 65 amino acid residues corresponding to the transmembrane and cytoplasmic domains of syndecan-2, was unable to pull down neurofibromin from rat brain extract (Fig. $1 B$ ). These biochemical results confirm an interaction between syndecan-2 and native neurofibromin and indicate that the transmembrane and cytoplasmic domains of syndecan are required for neurofibromin binding. GST-syndecan-2 also precipitated syndecan-3 from brain extracts (Fig. 1B), which is consistent with the known multimerization of syndecans via their transmembrane domains (Asundi and Carey, 1995).

\section{All four mammalian syndecans interact with neurofibromin}

Syndecans 1-4 are very similar to each other in their transmembrane and cytoplasmic domains (60-80\% amino acid identity) but share minimal homology in the extracellular regions (Fig. $2 A$ ). Syndecan-1, -3 , and -4 also interacted with neurofibromin in the yeast two-hybrid system (Fig. 2A). The constructs of syndecan-1, -3 , and -4 (fused to the GAL4 activation domain) were made to mimic the syndecan-2 cDNA isolated in the original yeast two-hybrid screen; they contained 58 residues of the extracellular domain and the entire transmembrane and cytoplasmic domains (Fig. 2A). Both $\mathrm{J} / \mathrm{Jn}$ and $\mathrm{P} / \mathrm{Pn}$ fragments of neurofibromin were able to interact with syndecans $1-4$ (Fig. $2 A$ ), although the interaction between Jn and syndecan-1 was relatively weak. The same fragments of neurofibromin did not interact with CASK (data not shown), confirming the specificity of the interaction.

\section{Both transmembrane and cytoplasmic domains of syndecans are involved in interaction with neurofibromin}

The GST pulldown result (see Fig. $1 B$ ) and the fact that all four syndecans bind to $\mathrm{J}$ and $\mathrm{P}$ regions of neurofibromin suggest that the neurofibromin binding site or sites lie within the highly conserved transmembrane or cytoplasmic regions of syndecans 1-4 (Fig. 2A). We first tested whether the cytoplasmic domain alone was sufficient for interaction with neurofibromin in yeast two-hybrid assays. A construct containing the cytoplasmic domain and four residues of the transmembrane domain of syndecan-2 (synd-2C) showed no interaction with either the Jn or Pn fragment of neurofibromin (Fig. 2B, second row). Synd-2C, however, also did not interact with CASK, despite an intact $\mathrm{C}$ terminus containing the CASK PDZ-binding sequence (-EFYA), suggesting that conformation of this fusion is incorrect. Similarly, synd$2 \Delta \mathrm{TM}$ (a construct deleted specifically for the transmembrane domain of syndecan-2) was unable to interact with either neurofibromin or CASK (Fig. 2B, third row). Because the transmembrane domains of syndecans mediate homo- and heteromultimerization of syndecans (Asundi and Carey, 1995) (Fig. 1B; data not shown), we suspect that association via the transmembrane domain is essential to present the cytoplasmic domain appropriately for interaction with neurofibromin and CASK.

The two most highly conserved regions in the cytoplasmic domains of syndecans are the C terminus (-EFYA) and a membrane-proximal segment (RMRKKDEGSY). A mutant lacking the last three amino acids of syndecan-2 (synd-2 $\Delta 3$ ) retained strong interaction with the $\mathrm{J} / \mathrm{Jn}$ and $\mathrm{P} / \mathrm{Pn}$ fragments of neurofibromin but lost its interaction with CASK (Fig. 2B). Thus neurofibromin binds to a region of syndecan distinct from the C-terminal CASK binding site. Deletion of the membraneproximal sequence RKKDEGSY (synd-2 $223-30$ ) virtually abolished syndecan interaction with either Jn and Pn fragments of neurofibromin (Fig. 2B), reducing the binding activities to 2 and $0.8 \%$ of wild-type, respectively (based on quantitative $\beta$-galactosidase assays). This result suggests that the conserved membrane-proximal sequence RKKDEGSY is indeed important for interaction with both regions of neurofibromin. We proceeded to construct double alanine substitution mutants to map the critical residues. Replacement of RK or KD with alanines (synd2RK/AA and synd-2KD/AA) greatly inhibited the interaction with either Jn or Pn fragments of neurofibromin (Fig. 2B). The effects were similar to that of the RKKDEGSY deletion mutant (synd-2 $\Delta 23-30$ ). Mutation of EG to AA (synd-2EG/AA) had a modest effect on the interaction with neurofibromin, retaining 34 and $85 \%$ activity for Jn and Pn fragments, respectively. Synd2SY/AA mutation had no effect on neurofibromin binding. Taken together, the sequence RKKD within the membrane-proximal region of syndecan appears to be most important for neurofibromin interaction.

We found, however, that other regions of the cytoplasmic domain of syndecan-2 also may be involved in the interaction with neurofibromin. Deletion of the sequence DLGERK (synd-2 $\Delta 17-$ 22) that lies immediately C-terminal to RKKDEGSY reduced the interactions with Jn and Pn fragments to 9 and 9.6\%, respectively (Fig. 2B). Deletion of the more C-terminal sequence YQKAPT (synd-2 $\Delta 6-11$ ) reduced the activities to $18.4 \%$ (for Jn) and $24.9 \%$ (for $\mathrm{Pn}$ ). These results suggest that sequences DLGERK and YQKAPT also contribute to the interaction with neurofibromin, although they are less important than the membrane-proximal RKKD motif. This is supported further by the results from $\mathrm{C}$-terminal truncation mutants. Deleting the last 11 amino acids of syndecan-2 (synd-2 $\Delta 11$ ) greatly impaired its ability to associate with neurofibromin (Fig. $2 B$ ); further deletion of $20 \mathrm{C}$-terminal amino acids (synd-2 $\Delta 20$ ) completely abolished neurofibromin binding (Fig. $2 B$ ).

\section{Developmental expression and biochemical fractionation of neurofibromin, syndecan, and CASK}

To study the biochemical interactions of neurofibromin, syndecans, and CASK in vivo, we first analyzed their expression patterns in rat brain by immunoblotting (Fig. 3). Expression of neurofibromin protein was highest in embryonic brain but remained at moderate levels throughout postnatal development (Fig. 3A). Syndecan-3 (also known as neuronal or N-syndecan) 


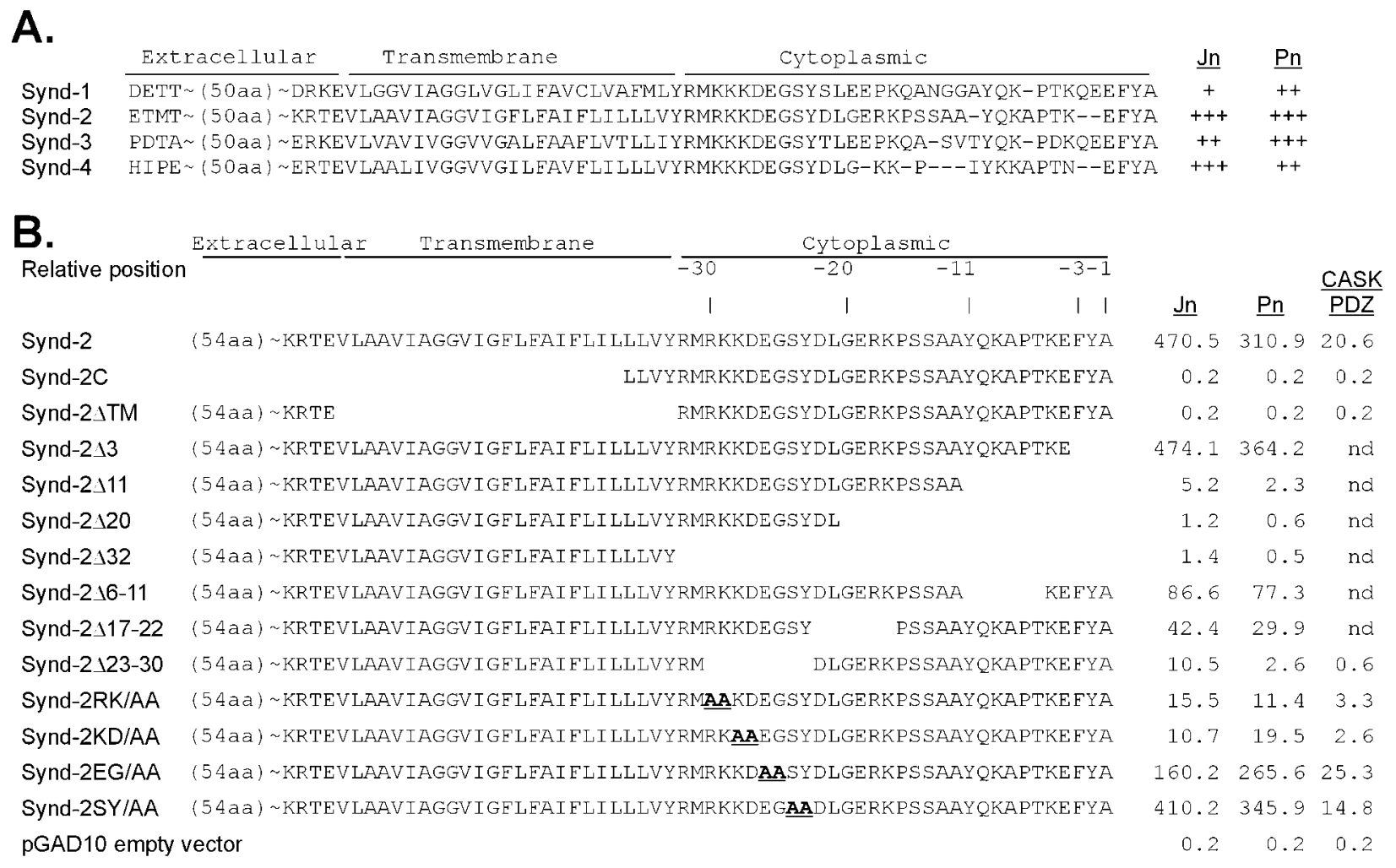

Figure 2. Yeast two-hybrid analysis of the neurofibromin-syndecan interaction. Yeast two-hybrid interactions of neurofibromin fragments Jn and Pn with syndecans 1-4 $(A)$ or with various syndecan-2 deletion and substitution mutants $(B)$ were determined by induction of the reporter gene $\beta$-galactosidase. $A$, The activity of $\beta$-galactosidase was measured by the time taken for colonies to turn blue in the $\mathrm{X}$-gal filter assay;,+++ 1 hr; ++ , 1-3 hr;,$+ 3-5 \mathrm{hr} ;-$, no significant reaction. $B, \beta$-galactosidase activity was measured by liquid culture assay. The numbers represent units of $\beta$-galactosidase activity (see Materials and Methods). Neurofibromin fragments were fused to the LexA DNA binding domain, and syndecans were fused to the GAL-4 transactivation domain. The sequences of syndecans in GAL-4 fusions are aligned, with deleted sequences left blank. Approximately 50 aa of the extracellular domain are omitted because of a lack of homology among different syndecans.

showed higher expression in embryonic and early postnatal rat brain than in adult brain (Fig. $3 A$ ). Because our syndecan-1 and -4 antibodies do not work efficiently on immunoblots of brain, where syndecans are modified highly and heterogeneously by heparan sulfate, we were unable to assess the levels of these proteins. CASK is expressed fairly constantly in embryonic and postnatal brain (Fig. $3 A$ ).

The subcellular distribution of neurofibromin, syndecan-3, and CASK was investigated by biochemical fractionation of adult rat brain (Fig. 3B). Neurofibromin is associated predominantly with membrane fractions, e.g., crude synaptosome (P2), light membrane (P3), lysed synaptosomal membrane (LP1), and crude synaptic vesicle (LP2) fractions. Syndecan-3 also is enriched in P2 and LP1. CASK is distributed more broadly in soluble and membrane fractions. Thus neurofibromin, syndecan-3, and CASK have nonidentical subcellular distributions overlapping in synaptosome (P2) and synaptosomal membrane (LP1) fractions.

To explore the possibility that neurofibromin is localized at synapses, we examined whether neurofibromin cofractionated with the postsynaptic density (PSD). Indeed, neurofibromin was present in both PSDI (extracted once with Triton X-100) and PSDII (extracted twice with Triton X-100) fractions; however, it was not enriched in the PSD like CASK or PSD-95 (Fig. 3C).

\section{Coimmunoprecipitation of neurofibromin, syndecan-3, and CASK from rat brain}

Because neurofibromin and CASK have different binding sites on syndecans, these three proteins may form a ternary protein com- plex. To test this in vivo, we performed coimmunoprecipitation experiments of rat brain extracts (Fig. 3D). Neurofibromin antibodies precipitated $>50 \%$ of neurofibromin from Triton X-100solubilized rat brain and coprecipitated $\sim 5-10 \%$ of syndecan-3. In addition, a small fraction of CASK was coimmunoprecipitated by neurofibromin antibody, indicating the existence of a native complex containing neurofibromin/syndecan/CASK. A control integral membrane protein, GluR2, was undetectable in neurofibromin immunoprecipitates, confirming the specificity of the coimmunoprecipitation.

\section{Overlapping distribution of neurofibromin and syndecan-3 at axons}

By immunofluorescence and immunogold EM localization, syndecan-2 is highly enriched at synapses (Hsueh et al., 1998), whereas syndecan-3 is concentrated on axons (Fig. $4 B$; Hsueh and Sheng, 1999). Immunostaining of immature cortical neurons in culture (7 DIV) showed neurofibromin to be distributed widely in neurons, including axons, dendrites, and cell bodies (Fig. 4A1; data not shown). In mature hippocampal neuronal cultures (20 DIV) neurofibromin antibody revealed staining of both puncta and long processes as well as diffuse cell body labeling (Fig. $5 A 1, B 1)$. By confocal microscopy, neurofibromin staining overlapped with syndecan-3 in axonal processes of both young and mature neuronal culture (Figs. $4 E, 5 A$ ), whereas in mature hippocampal neurons it colocalized with syndecan-2 in a punctate synaptic pattern (Fig. $5 B$ ).

We also performed double immunofluorescence staining of rat 

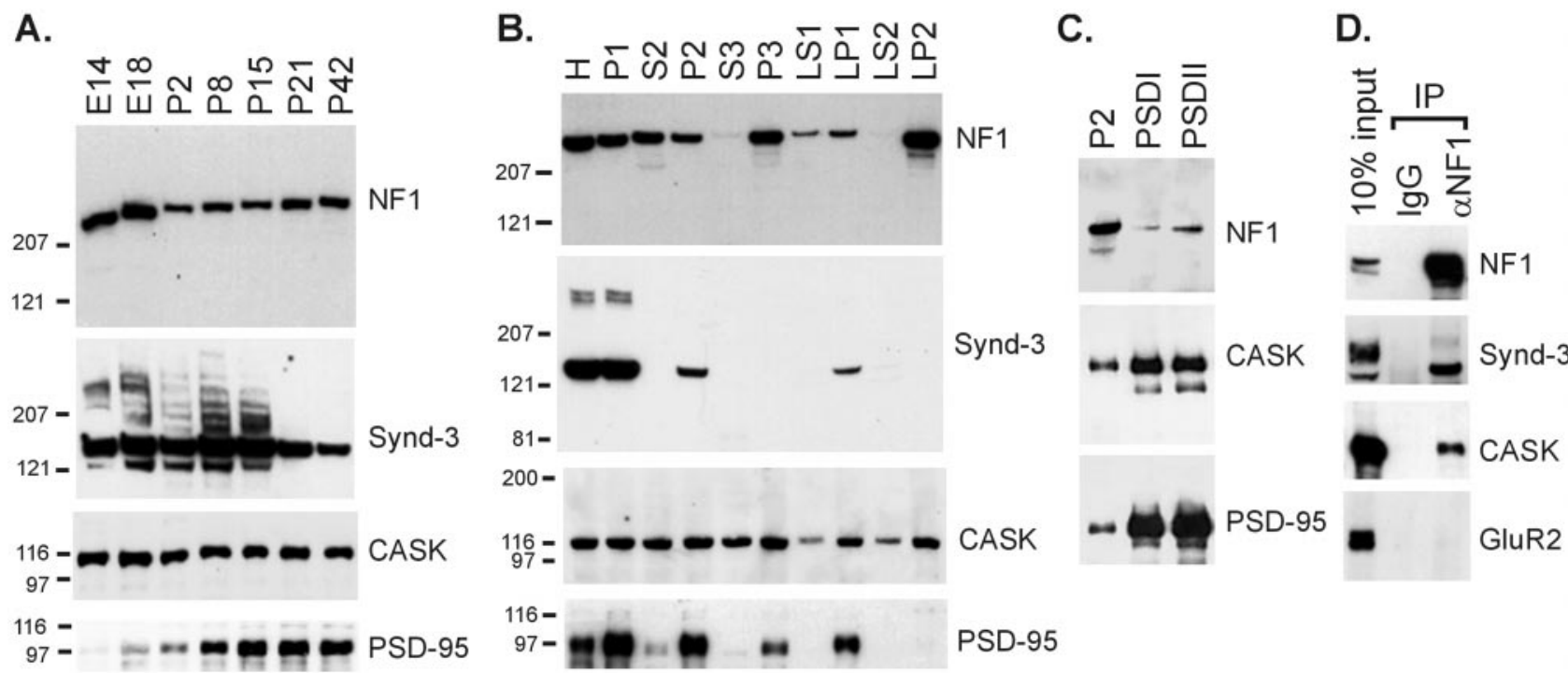

Figure 3. Expression, fractionation, and coimmunoprecipitation of neurofibromin, syndecan-3, and CASK. $A$, Developmental expression profile. Crude synaptosomal membrane fractions were collected from rat whole brain at different embryonic $(E)$ and postnatal $(P)$ ages $($ days) and immunoblotted for the indicated proteins. $B$, Biochemical fractionation. Adult rat brain homogenate was fractionated by centrifugation (see details in Materials and Methods). The resulting fractions were immunoblotted for the indicated proteins. $H$, Total homogenate; $P 1$, crude nuclear pellet; $S 2$, supernatant of P2 pellet; $P 2$, crude synaptosomal membrane; $S 3$, cytosolic fraction; $P 3$, light membrane fraction; $L S 1$, supernatant of LP1; $L P 1$, lysed synaptosomal fraction; $L S 2$, supernatant of LP2; $L P 2$, crude synaptic vesicle fraction. $C$, Presence of neurofibromin in the postsynaptic density $(P S D)$ fraction of adult rat brain. Equal protein amounts of crude synaptosomal fraction (P2), PSD fraction I (PSDI; extracted once by Triton X-100), and fraction II (PSDII; extracted twice by Triton X-100) were immunoblotted for neurofibromin, CASK, and PSD-95. D, Coimmunoprecipitation of neurofibromin, syndecan, and CASK. Triton X-100-solubilized total homogenate of P7 rat brain was immunoprecipitated with neurofibromin antibodies or control rabbit IgG. The precipitates were analyzed by immunoblotting for neurofibromin, syndecan-3, CASK, and GluR2.

brain sections. In concordance with the hippocampal culture results, neurofibromin colocalized with syndecan-3 in axonal pathways such as the corpus callosum (Fig. 6A). Neurofibromin also codistributed with syndecan- 2 in synapses in the mossy fiber tract of the hippocampus (Fig. 6B). Taken together with staining results of cultured neurons, neurofibromin localization overlaps with different syndecans in different subcellular compartments, namely with syndecan-2 at synaptic sites and with syndecan-3 in axons.

\section{DISCUSSION}

\section{Interaction between neurofibromin and the syndecans}

To date the only identified biochemical function of neurofibromin is that of stimulating the GTPase activity of Ras. Here, we report a novel interaction between neurofibromin and the syndecan family of transmembrane HSPGs. To our knowledge this represents the first demonstration of a neurofibromin-interacting protein other than Ras.

Neurofibromin and the syndecans are expressed widely in both neurons and glia (Daston et al., 1992; Nordlund et al., 1993; Hsueh et al., 1998; Hsueh and Sheng, 1999). Syndecans 1-4 show differential cell type-specific patterns of expression in the rat CNS (Hsueh and Sheng, 1999). For instance, syndecan-2 and -3 are mainly neuronal, whereas syndecan-4 is expressed selectively in glial cells. Syndecans also are distributed differentially with respect to subcellular compartment, syndecan-2 being concentrated in synapses (Hsueh et al., 1998) and syndecan-3 in axons (Hsueh and Sheng, 1999). The functional significance of this differential localization is not clear. However, because neurofibromin can bind to all four known mammalian syndecans, it may associate with different syndecan family members in different cell types or at different subcellular locations in the same way that CASK can
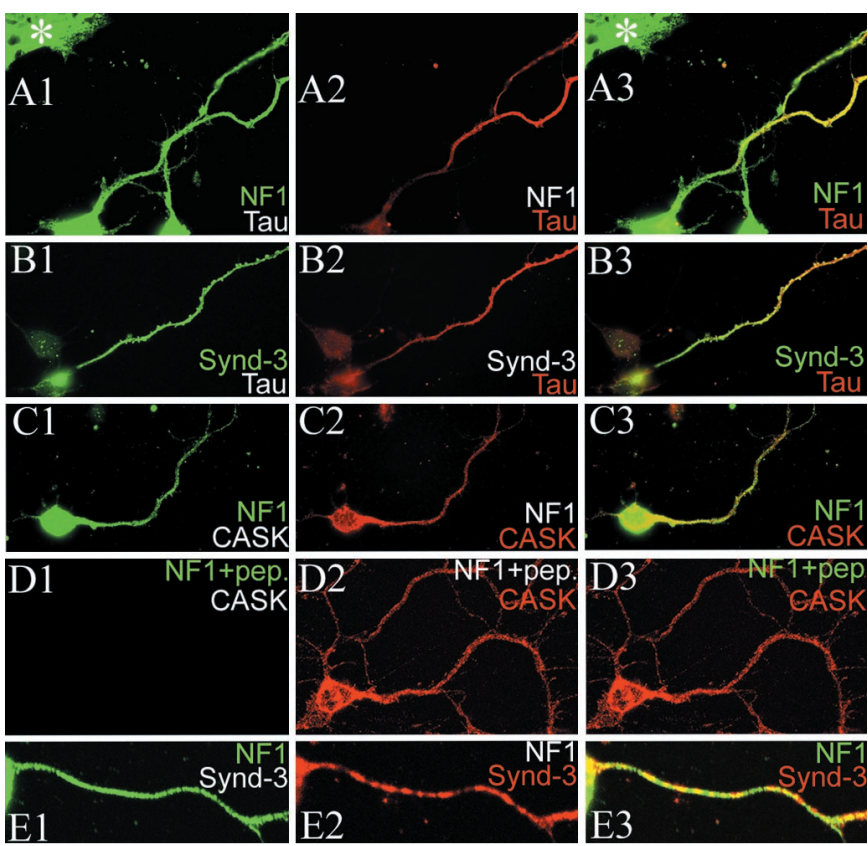

Figure 4. Overlapping distribution of neurofibromin, syndecan-3, and CASK in axons of 7 DIV cultured cortical neurons. $A-C$, Indirect double immunofluorescence staining was performed with neurofibromin (NF1), syndecan-3 (Synd-3), CASK, and tau antibodies. Each group of images represents the same field visualized for the protein indicated in color in each panel. Tau is used as an axonal marker. $D$, Neurofibromin antibody was preincubated with antigenic peptide for $1 \mathrm{hr}$ before being added to the cells. The neurofibromin staining signal was inhibited completely by competition of antigenic peptide, suggesting specificity of staining. $E$, Confocal images of double immunofluorescence staining with the use of the TSA fluorescence system (see details in Materials and Methods). Asterisks in $A$ mark neurofibromin-positive glial cells at top left corner. 

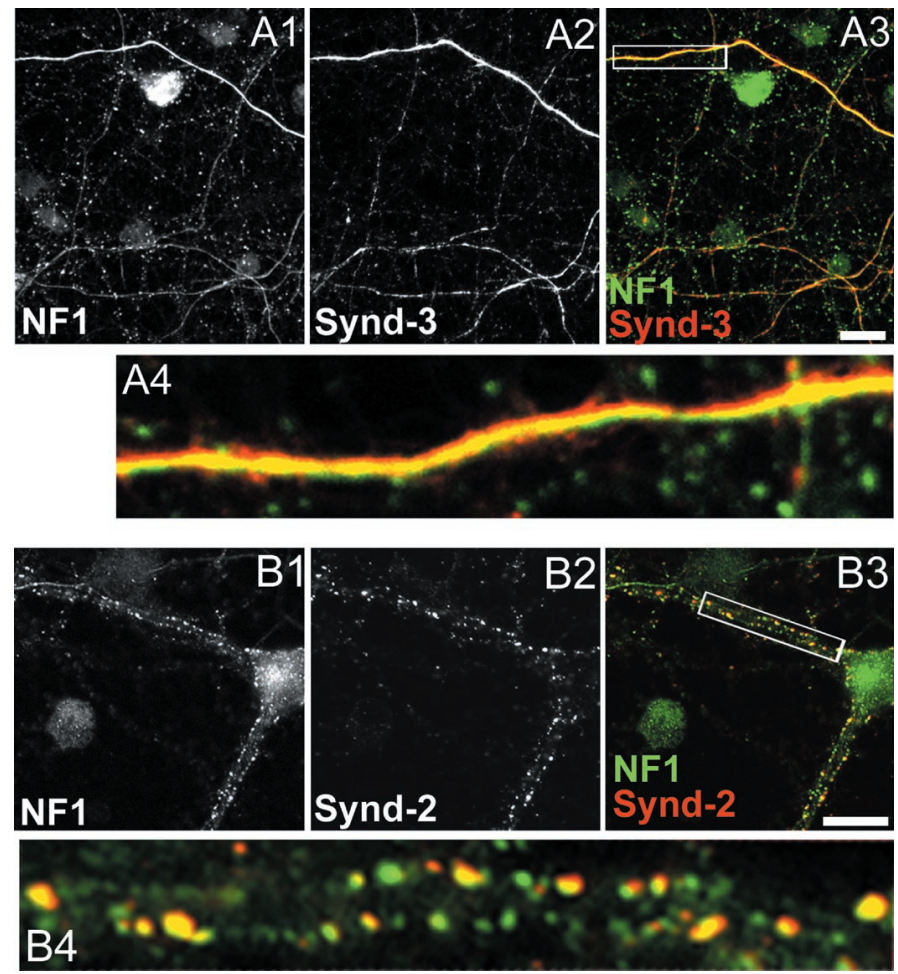

Figure 5. Colocalization of neurofibromin with different syndecans at different subcellular compartments in mature hippocampal neuronal cultures. Confocal analysis of indirect immunofluorescence staining of 20 DIV hippocampal neuronal cultures was performed. Each group of images represents the same field visualized for the protein indicated in color in each panel. The fourth panel of each group is a higher magnification image of the inset in the third panel. A, Double staining of neurofibromin and syndecan-3. $B$, Double staining of neurofibromin and syndecan-2. TSA fluorescence system was used for syndecan- 2 and syndecan-3 staining (see details in Materials and Methods).

interact with multiple distinct syndecans (Hsueh et al., 1998, 1999).

Remarkably, two widely separated regions in neurofibromin ( $\mathrm{J}$ and P) can bind to syndecan. This bipartite interaction could increase the avidity of binding between neurofibromin and syndecan proteins. By yeast two-hybrid analysis that used various mutants of syndecan-2, we could not distinguish between the binding of $\mathrm{J}$ and $\mathrm{P}$ fragments, suggesting that the $\mathrm{J}$ and $\mathrm{P}$ fragments may bind to the same or overlapping regions of syndecan. Surprisingly, there is no sequence similarity between the $\mathrm{J}$ and $\mathrm{P}$ regions. It will be interesting to know whether the $\mathrm{J}$ and $\mathrm{P}$ segments lie close to each other in the tertiary or quaternary structure of neurofibromin and whether they compete or collaborate with each other for binding to syndecan.

Deletion analysis indicates that both transmembrane and cytoplasmic domains of syndecan are required for interaction with neurofibromin. The transmembrane domain of syndecan is unlikely to be the direct binding site of neurofibromin and previously has been shown to act as a multimerization domain. Because the cytoplasmic tail of syndecan is short ( $\sim 30$ amino acids), it is possible that multimerization via the transmembrane region is required for its presentation in the correct conformation or stoichiometry for binding to neurofibromin. For instance, neurofibromin binding may require two or more cytoplasmic tails of syndecans in close proximity.
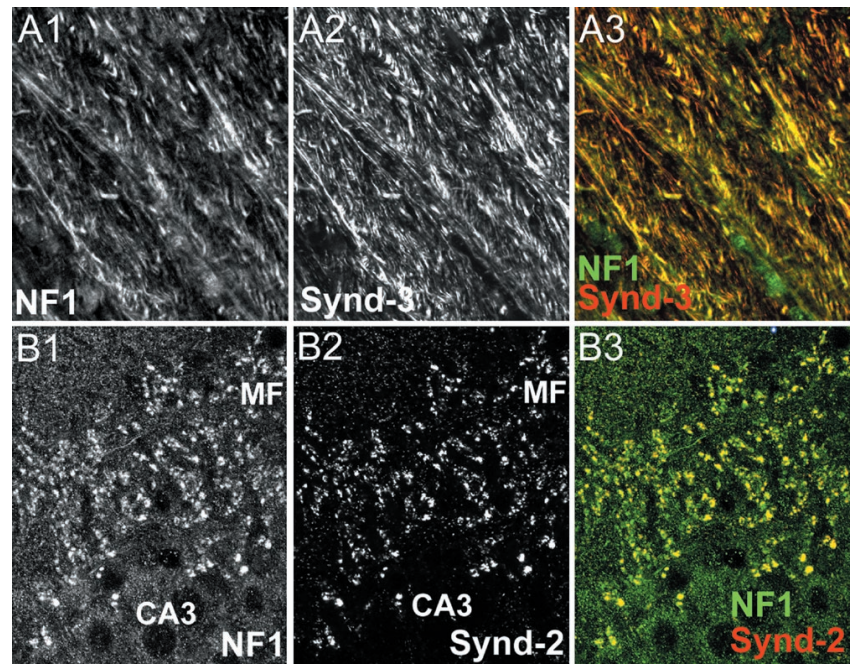

Figure 6. Colocalization of neurofibromin with different syndecans in different regions of rat brains. $A$, Double staining with neurofibromin and syndecan-3 in corpus callosum. $B$, Double staining with neurofibromin and syndecan-2 in region CA3 of hippocampus. $M F$, Mossy fiber tract. Each group of images represents the same field visualized for the protein indicated in color in each panel. TSA fluorescence system was used for syndecan-2 and syndecan-3 staining (see details in Materials and Methods).

\section{Role of the neurofibromin-syndecan interaction}

The biochemical fractionation studies indicate that neurofibromin is associated predominantly with brain membrane fractions (see Fig. 3B). Binding to the transmembrane syndecan proteins provides a potential mechanism for the membrane association of neurofibromin. Syndecans are targeted to specific subcellular sites where they probably are anchored via $\mathrm{C}$ terminus-PDZ domain interaction to scaffold proteins such as CASK (Cohen et al., 1998; Hsueh et al., 1998) and syntenin (Grootjans et al., 1997). PDZcontaining scaffolds typically assemble large protein complexes at particular sites at the plasma membrane (Sheng and Sala, 2001). CASK also binds to additional proteins such as Veli/LIN-7 and Mint1/LIN-10, which in turn interact with other proteins (Bredt, 1998). The binding of neurofibromin to syndecan therefore could target it to a specialized molecular microdomain organized by CASK and associated proteins at the cell surface.

What is the functional significance of the interaction between neurofibromin and the syndecans? The exact roles of syndecan HSPGs at the cell surface are not clearly understood. In general, cell surface HSPGs are believed to play roles in cell adhesion and intercellular signaling, often acting as binding sites for growth/ differentiation factors and as coreceptors for conventional receptors (Carey, 1997; Perrimon and Bernfield, 2000). Syndecans are implicated in the maintenance of differentiation and in suppression of tumor growth in epithelial cells (Leppa et al., 1992). In neurons, syndecan-2 promotes the development of dendritic spines (Ethell and Yamaguchi, 1999). By association with syndecan-2, neurofibromin may contribute to synaptic RasGAP activity. All of these activities are pertinent to cell differentiation and proliferation and/or to synaptic plasticity and hence are relevant to the tumorigenic and learning defect phenotypes of NF1. The association of neurofibromin with syndecans and CASK suggests that it may function in an adhesion-signaling complex at the cell surface, perhaps via the targeting of neurofibromin RasGAP activity to syndecan-containing microdomains. 


\section{REFERENCES}

Asundi V, Carey D (1995) Self-association of N-syndecan (syndecan-3) core protein is mediated by a novel structural motif in the transmembrane domain and ectodomain flanking region. J Biol Chem 270:26404-26410.

Ballester R, Marchuk D, Boguski M, Saulino A, Letcher R, Wigler M, Collins F (1990) The NF1 locus encodes a protein functionally related to mammalian GAP and yeast IRA proteins. Cell 63:851-859.

Bernfield M, Kokenyesi R, Kato M, Hinkes MT, Spring J, Gallo RL, Lose EJ (1992) Biology of the syndecans: a family of transmembrane heparan sulfate proteoglycans. Annu Rev Cell Biol 8:365-393.

Brannan C, Perkins A, Vogel K, Ratner N, Nordlund M, Reid S, Buchberg A, Jenkins N, Parada L, Copeland N (1994) Targeted disruption of the neurofibromatosis type- 1 gene leads to developmental abnormalities in heart and various neural crest-derived tissues. Genes Dev 8:1019-1029.

Bredt DS (1998) Sorting out genes that regulate epithelial and neuronal polarity. Cell 94:691-694.

Butz S, Okamoto M, Südhof TC (1998) A tripartite protein complex with the potential to couple synaptic vesicle exocytosis to cell adhesion in brain. Cell 94:773-782.

Carey D (1997) Syndecans: multifunctional cell-surface coreceptors. Biochem J 327:1-16.

Cichowski K, Shih T, Schmitt E, Santiago S, Reilly K, McLaughlin M, Bronson R, Jacks T (1999) Mouse models of tumor development in neurofibromatosis type 1 . Science 286:2172-2176.

Cohen AR, Woods DF, Marfatia SM, Walther Z, Chishti AH, Anderson JM (1998) Human Cask/Lin-2 binds syndecan-2 and protein 4.1 and localizes to the basolateral membrane of epithelial cells. J Cell Biol 142:129-138.

Daston M, Scrable H, Nordlund M, Sturbaum A, Nissen L, Ratner N (1992) The protein product of the neurofibromatosis type 1 gene is expressed at highest abundance in neurons, Schwann cells, and oligodendrocytes. Neuron 8:415-428.

David G (1993) Integral membrane heparan sulfate proteoglycans. FASEB J 7:1023-1030.

Ethell I, Yamaguchi Y (1999) Cell surface heparan sulfate proteoglycan syndecan-2 induces the maturation of dendritic spines in rat hippocampal neurons. J Cell Biol 144:575-586.

Grootjans J, Zimmermann P, Reekmans G, Smets A, Degeest G, Durr J, David G (1997) Syntenin, a PDZ protein that binds syndecan cytoplasmic domains. Proc Natl Acad Sci USA 94:13683-13688.

Guo H, The I, Hannan F, Bernards A, Zhong Y (1997) Requirement of Drosophila NF1 for activation of adenylyl cyclase by PACAP38-like neuropeptides. Science 276:795-798.

Guo H, Tong J, Hannan F, Luo L, Zhong Y (2000) A neurofibro- matosis-1-regulated pathway is required for learning in Drosophila. Nature 403:895-898.

Hsueh Y-P, Sheng M (1999) Regulated expression and subcellular localization of syndecan heparan sulfate proteoglycans and the syndecanbinding protein CASK/LIN-2 during rat brain development. J Neurosci 19:7415-7425.

Hsueh Y-P, Yang F-C, Kharazia V, Naisbitt S, Cohen AR, Weinberg RJ, Sheng M (1998) Direct interaction of CASK/LIN-2 and syndecan heparan sulfate proteoglycan and their overlapping distribution in neuronal synapses. J Cell Biol 142:139-151.

Huttner W, Schiebler W, Greengard P, DeCamilli P (1983) Synapsin I (protein I), a nerve terminal-specific phosphoprotein. III. Its association with synaptic vesicles studied in a highly purified synaptic vesicle preparation. J Cell Biol 5:1374-1388.

Jacks T, Shih T, Schmitt E, Bronson R, Bernards A, Weinberg R (1994) Tumour predisposition in mice heterozygous for a targeted mutation in NF1. Nat Genet 7:353-361.

Kaech SM, Whitfield CW, Kim SK (1998) The LIN-2/LIN-7/LIN-10 complex mediates basolateral membrane localization of the $C$. elegans EGF receptor LET-23 in vulval epithelial cells. Cell 94:761-771.

Kim SK (1997) Polarized signaling: basolateral receptor localization in epithelial cells by PDZ-containing proteins. Curr Opin Cell Biol 9:853-859.

Leppa S, Mali M, Miettinen H, Jalkanen M (1992) Syndecan expression regulates cell morphology and growth of mouse mammary epithelial tumor cells. Proc Natl Acad Sci USA 89:932-936.

Martin G, Viskochil D, Bollag G, McCabe P, Crosier W, Haubruck H, Conroy L, Clark R, O'Connell P, Cawthon R (1990) The GAPrelated domain of the neurofibromatosis type 1 gene product interacts with Ras p21. Cell 63:843-849.

Niethammer M, Sheng M (1998) Identification of ion channel-associated proteins using the yeast two-hybrid system. In: Methods in enzymology (Conn PM, ed), pp 104-122. New York: Academic.

Nordlund M, Gu X, Shipley M, Ratner N (1993) Neurofibromin is enriched in the endoplasmic reticulum of CNS neurons. J Neurosci 13:1588-1600.

Perrimon N, Bernfield M (2000) Specificities of heparan sulfate proteoglycans in developmental processes. Nature 404:725-728.

Schlessinger J, Lax I, Lemmon M (1995) Regulation of growth factor activation by proteoglycans: what is the role of the low affinity receptors? Cell 83:357-360.

Sheng M, Sala C (2001) PDZ domains and the organization of supramolecular complexes. Annu Rev Neurosci 24:1-29.

Vogel K, Klesse L, Velasco-Miguel S, Meyers K, Rushing E, Parada L (1999) Mouse tumor model for neurofibromatosis type 1. Science 286:2176-2179. 